\title{
Lithium in globular clusters
}

\author{
Andreas J. Korn \\ Dept. of Physics and Astronomy, Uppsala University, \\ Box 516, 75120 Uppsala, Sweden \\ email: andreas.korn@fysast.uu.se
}

\begin{abstract}
I review recent progress in mapping out and understanding the behaviour of stellar surface abundances of lithium as evidenced by spectroscopic studies of nearby globular clusters (GCs). It will become clear that these observations necessitate revisions to the canonical picture of stellar and globular-cluster evolution: stars evolve with additional non-convective mixing processes and GCs are not simple stellar populations. In spite of these complications, GCs are excellent test beds for chemical-abundance studies. Spectroscopic observations of GC stars of different evolutionary stages reveal systematic trends of surface abundances likely caused by atomic diffusion and mixing. Correcting for their combined effect on surface lithium, a stellar solution to the cosmological lithium discrepancy is likely, if not probable. However, a definitive answer can only be given once we know the effective temperatures of warm subdwarfs and subgiants to high accuracy and understand the processes which give rise to the mixing needed to moderate atomic diffusion.
\end{abstract}

Keywords. Stars: Population II, atmospheres, abundances - diffusion - line: formation - globular clusters: individual (M 92, NGC 6397, NGC 6752) - cosmology: early universe - techniques: spectroscopic

\section{Introduction}

Cosmology with stars. This was the implicit promise of the seminal discovery of a uniform lithium abundance among warm halo field stars in 1982 by Monique and Francois Spite (Spite \& Spite 1982). A quarter century on, in the era of precision cosmology (PC), heralded by balloon experiments like MAXIMA and BOOMERANG and fully established through the WMAP-satellite all-sky measurements, we realize that the evolution of stellar-surface lithium abundances is more complicated and the inference of its primordial (or Big-Bang nucleosynthesis, BBN) value less direct. As early as 1984, Michaud, Fontaine \& Beaudet (1984) cautioned that the Spite plateau of lithium was unlikely to represent the unaltered primordial abundance of lithium, as diffusive processes inside the stars would inevitably deplete the stellar surface layers of heavy elements over the course of the billion-year stellar lifetimes.

It is the intention of this review article to give an overview of the latter half of this time frame of Galactic lithium studies with potential cosmological implications. This is the period when the current generation of $8-10 \mathrm{~m}$ telescopes became available giving routine access to much fainter Spite-plateau stars. Apart from singular heroic efforts with 4mclass telescopes (e.g. Pasquini \& Molaro 1996), lithium in globular clusters (GCs) has been exclusively studied with efficient spectrographs on Keck, the VLT and their siblings. The TOP (turn-off point) stars in even the most nearby low-reddening GCs (e.g. NGC 6397) are no brighter than $V \approx 16.5^{\mathrm{m}}$. In the very metal-poor GC M 92, the TOP stars have $V \approx 18.5^{m}$ which makes studies at high resolution $(R \approx 40000)$ and high signal-to-noise ratio $(\mathrm{S} / \mathrm{N} \approx 100)$ a true challenge, even with $50+$ square metres of light-collecting area and $90 \%$ overall quantum efficiency. 
For the sake of a historical assessment, I subdivide the roundabout 12 years to be covered here into two periods: the first six years (1998-2003, Sect. 2) are characterized by a single-star observational approach and small-number statistics, with in part contradicting results; the second six years (2004-2009, Sect. 3) have led to some far-reaching revisions based on multi-object spectroscopy coupled with better observations and better modelling. Some apparent contradictions are discussed. Work in progress is presented in Sect. 4 and concluding remarks are given in Sect. 5 .

\section{The HIRES and UVES years (1998-2003)}

Scientists with access to Keck had a head start to the GC lithium business. Building on observations of Deliyannis, Boesgaard \& King (1995), Boesgaard et al. (1998) analysed high-resolution, moderate-S/N spectra of a handful of subgiants in M 92 and concluded that there is a significant dispersion in lithium abundances among otherwise very similar stars. This dispersion was interpreted to be an indication for different levels of lithium depletion taking place in these stars, possibly correlated with the stars' angular-momentum history. This is not necessarily a far-fetched thought (cf. Pinsonneault, these proceedings). But to make sense of a marked dispersion in M 92 on the one hand and no dispersion (plus a few outliers) on the field-star Spite plateau would require to postulate very different angular-momentum conditions for these two groups of stars.

Bonifacio (2002) subsequently analysed the same M 92 data and concluded that there is no compelling evidence for dispersion beyond the level expected from observational uncertainties. Somewhat surprisingly, no one has to this day reinvestigated the chemical signatures of little evolved stars in M 92 (but see Sect. 4).

Across the Atlantic, a team of European researchers used large amounts of observing time at the VLT to systematically explore the nucleosynthesis within a variety of GCs (cf. Bragaglia, these proceedings, for an overview). One of the early targets was the smallish GC NGC 6397. Bonifacio et al. (2002) studied lithium in a dozen TOP stars in this GC and arrived at a common lithium abundance of $\log \varepsilon(\mathrm{Li})=2.34 \pm 0.06$ on the customary $\operatorname{logarithmic}$ scale that equates $\log \varepsilon(\mathrm{H})$ with 12 . It is probably fair to say that in the early years of this century it looked as if the GC Spite plateau behaved just like that of the field stars.

This idea was overthrown by Pasquini et al. (2005) who observed a 0.45 dex tip-to-tip dispersion among nine TOP stars in NGC 6752 at $[\mathrm{Fe} / \mathrm{H}]=-1.5$. They went beyond Boesgaard and collaborators in showing that lithium in this cluster correlates with other elements: sodium, oxygen and nitrogen. Together with other inverse correlations traced down to the main-sequence TOP (O-Na, Mg-Al, Gratton et al. 2001), it thus became clear that a significant fraction of the GC stars we observe today suffer from intra-cluster pollution through a previous generation of (more massive) GC stars. This paradigm shift in GC research paved the way to research on multiple populations in GCs (Piotto et al. 2007, Lee et al. 2009).

\section{The FLAMES years (2004-2009)}

This period was characterized by several breakthroughs, theoretical and observational ones alike. It became clear that primordial lithium as predicted by standard WMAPcalibrated BBN and the Spite-plateau lithium abundance are irreconcilable at face value, the difference exceeding $0.3 \mathrm{dex}$ (Coc et al. 2004). Building on 20 years of work within the Michaud school, Richard, Michaud \& Richer (2005) tuned their stellar-structure models with additional mixing and proposed a stellar-physics solution: the surface lithium 
diffuses out of the convective envelope according to the physical laws for inhomogeneous gases in a gravitational field, but this gravitational settling is moderated by an unspecified parametrized mixing process below the convective envelope. It is this (turbulent) mixing that manages to keep the Spite plateau thin and flat, even though the surface abundance is reduced by as much as 0.4 dex. Since this postulated mixing lacks a physical interpretation, the models can be said to loose their predictive power. But it turns out that only a subset of models fulfills the observational constraints: in the language of mixing $\dagger$ this is the range from T6.0 to T6.28.

I started to look into the same issue from an observational point as early as 2003 . With the vagaries of Paranal weather, it took two years to collect spectroscopic data of sufficient quality to take a fresh look at chemical abundances in NGC 6397. But what a data set it turned out to be! With the advances in instrumentation at the VLT, we not only got high-resolution data for 18 stars along the evolutionary sequence of NGC 6397 with UVES in fibre mode; we also got $100+$ spectra of intermediate resolution $(R=25,000)$ using FLAMES-MEDUSA. This instrument has really propelled us into a new era of GC research!

In a series of papers, Korn et al. (2006, 2007) and Lind et al. (2008) showed that there are systematic trends of surface abundances with evolutionary stage. The TOP stars show systematically lower abundances (by up to $0.2 \mathrm{dex}$ ) than the RGB stars. This is indeed what one would expect from gravitational settling: it is most efficient when the convective envelope (in itself always fully mixed on a convective-turnover time scale) is thinnest and least massive. Once the stars reach the RGB, the convective envelope expands inward and mixes the settled elements back to the surface. With a notable exception.

The run of surface lithium from the TOP to the RGB is instead characterized by a steep surface dilution. Lithium that settles into layers whose temperature exceeds 2 million Kelvin will capture a proton and disintegrate into $\mathrm{He}-4$ and $\mathrm{He}-3$, this lithium is thus lost and cannot replenish the surface abundance. But before the surface dilution sets is, we can see that lithium did actually settle: in the middle of the subgiant branch (SGB), the lithium abundance is higher than among TOP stars. We can understand why by looking at the structure of our model star which apart from the convective envelope has a region below the convection zone that is rich in lithium. This region is created by the mixing we introduced into the model. When the convection zone expands inward, it first encounters this region and brings its lithium content to the surface, the surface lithium abundance increases a little. Indeed, this is seen as $\mathrm{a} \approx 0.1$ dex abundance difference in lithium between TOP and SGB stars.

Lind et al. (2009) took this work further by analysing several hundred NGC 6397 cluster members with FLAMES-MEDUSA, from the main sequence to beyond the RGB bump. The derived run of lithium as a function of evolution is a textbook result, clearly demonstrating the accuracy with which such work can nowadays be executed. The upturn in the middle of the SGB discussed above and the need for extra mixing around the bump (at $M_{V}=0$ for NGC 6397) is seen very clearly. This extra mixing is now believed to be connected to a thermohaline instability that develops in connection with a mean-molecular-weight inversion (Charbonnel \& Zahn 2007). We really need more work along these lines, ideally coupling different elements, to verify that we understand all the

$\dagger$ There are, in principle, two parameters one can tune: the strengths and the density dependence of mixing. Richard, Michaud \& Richer (2005) chose to vary the strength and keep the run with density $\rho$ constant, with an exponent of -3 . The Tx.x notation then specifies the logarithm of the temperature $T_{0}$ at which the diffusion coefficient for mixing $D_{T}$ is connected to the atomic-diffusion coefficient for $\mathrm{He} D_{\mathrm{He}}$ according to $D_{T}=400 D_{\mathrm{He}}\left(T_{0}\right)\left(\rho / \rho\left(T_{0}\right)\right)^{-3}$. 


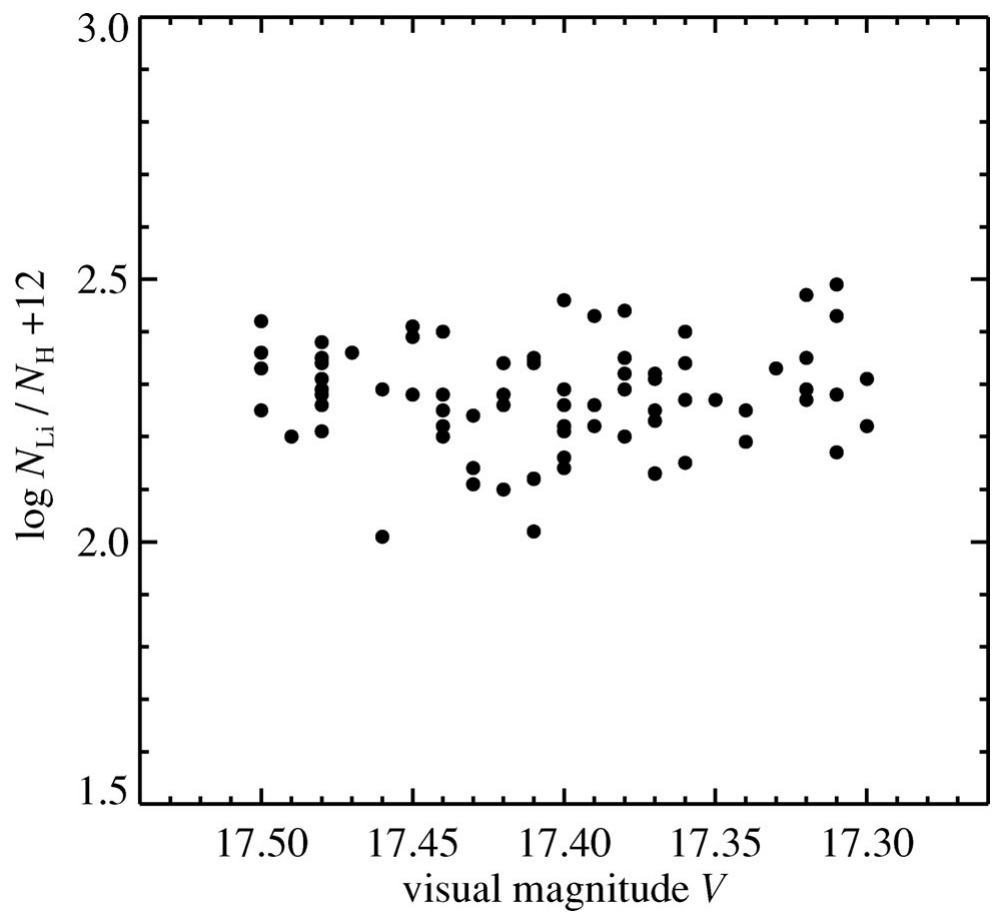

Figure 1. In the subgiant sample of González Hernández et al. (2009), there is little of a correlation between $\log \varepsilon(\mathrm{Li})$ and $V$ magnitude. If anything, there is a slight rise towards the middle of the SGB (brighter stars), like in the other works that studied NGC 6397.

variables at play here. For lithium production in more advanced stages of evolution, the reader is referred to Smith (these proceedings).

Finally, González Hernández et al. (2009) performed a vertical diffusion study comparing the lithium abundances between dwarfs and subgiants at a given $(B-V)$. Like Lind et al. (2009), they find a systematic difference between dwarfs and subgiants with the latter being 0.1 dex more lithium-rich. Working on a newly established effective-temperature scale (Balmer lines in 3D model atmospheres), they derive abundances as high as 2.4 dex for the subgiants. The also find trends with effective temperature that may or may not be significant (since this project was conceived as a vertical study, the $T_{\text {eff }}$ range covered is not very large and does not include the TOP; cf. Lind, these proceedings). Without resorting to statistical tests, there seems to be little of a positive trend of $\log \varepsilon(\mathrm{Li})$ with $V$ in Fig. 1. It would be good to see González Hernández and collaborators perform their analysis on all spectroscopically observed stars in NGC 6397 and include other elements into the picture.

\section{Ongoing analyses}

New evidence in favour of and against atomic diffusion of lithium was presented at IAU 268. Meléndez showed how the field-star Spite plateau can be reconciled with WMAPcalibrated BBN predictions using a T6.25 diffusion-and-mixing model à la Richard. When plotted versus stellar mass, there is a specific morphology that is well-captured by the model predictions. As a cautionary note, it should be said that a single such model (one for $[\mathrm{Fe} / \mathrm{H}]=-2.3$ ) was used to explain lithium abundances over a large range of metallicities. This should be remedied. Bonifacio presented challenging observations of 


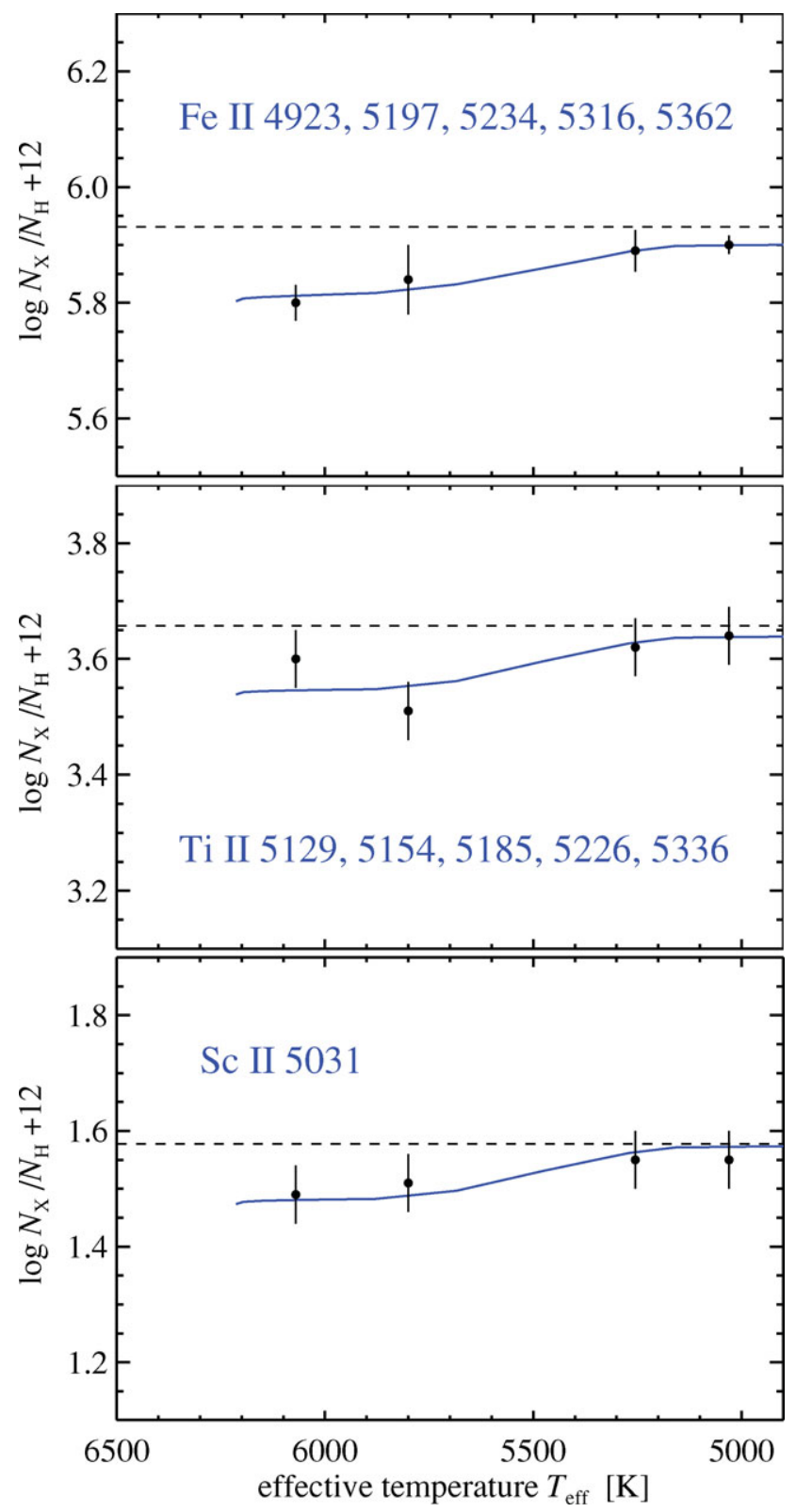

Figure 2. Measured abundance trends for stars in NGC $6752([\mathrm{Fe} / \mathrm{H}]=-1.6)$ versus predictions from a T6.2 Richard model. For iron, the star-to-star scatter in each group is given as an error bar, for titanium and scandium which are both measured on the group-averaged spectra, 0.05 dex is assumed.

Spite-plateau stars drawn from various populations in $\omega$ Cen. Identifying a common Spite plateau for the majority of these stars, he claimed that this must represent an unaltered stellar-birth value, as these different populations have different ages and should thus be affected differently by a temporal process like atomic diffusion. But as long as we do not know the age difference with certainty (and independent of the unknown helium-content difference), this argument rests on weak foundations. 
The ADiOS (Atomic Diffusion in Old Stars) team has begun to analyse VLT/FLAMESUVES data on NGC $6752([\mathrm{Fe} / \mathrm{H}]=-1.6)$ taken last year. The TOP stars in this cluster are faint $\left(V \approx 17.2^{m}\right)$ requiring exposure times in excess of $30 \mathrm{~h}$. So far, following a strict quality approach, only lines sensitive to surface gravity have been looked at: Fe II, Ti II and Sc II. Surface-gravity differences between stars can be inferred with high precision based on photometry and estimates of stellar mass and bolometric correction. In addition, both NLTE and 3D corrections are expected to be small for these lines, as they connect levels in the dominant ionization stage and form deep in the atmosphere. The abundance difference between the TOP and RGB stars is found to be $\Delta[\mathrm{Fe} / \mathrm{H}]=-0.10 \pm 0.03$ where the error is the star-to-star scatter in each group propagated into the abundance difference. Similar trends are found for titanium and scandium and all are in good agreement with T6.2 model predictions for this metallicity. With an atomic-diffusion correction of 0.25 dex for Li-7 and face-value surface abundances as high as $\log \varepsilon(\mathrm{Li})=2.5$, the primordial lithium abundance can be reached without much ado. Admittedly, it is towards lower metallicities where this game becomes more difficult to play. But beyond the metallicity of M 92 (for which new data was taken with HIRES on Keck last year (PI Cohen), analysis pending) this is not the realm of GC studies.

\section{Concluding remarks}

Studies of lithium in GCs have led to a number of important discoveries in recent years:

- Apart from intra-cluster Li-Na inverse correlations mainly affecting massive GCs, the behaviour of lithium in single GC stars resembles that of single field stars of the same mass range. This is to be expected assuming that the spin-down of metal-poor stars is fast, i.e. all stars evolve in a homologous manner irrespective of their initial angular momentum (which may well depend on environment).

- Higher surface lithium abundances among warm subgiants are indicative of dredgeup of lithium stored below the convective envelope. This is the most direct signature of atomic diffusion and mixing in such stars. In the presence of a strong Li-Na inverse correlation, atomic diffusion can still be studied using heavier elements.

- Extra mixing around the RGB bump sets in at a well-defined absolute magnitude $\left(M_{V}=0\right.$ for NGC 6397, Lind et al. 2009). Thermohaline mixing is now believed to be the main process responsible. A systematic investigation of how well the best stellarstructure models capture, e.g., the metallicity dependence of this extra mixing is still lacking.

What is almost completely absent from GC (and halo-star) studies so far is insight into rotation, mass loss and magnetic properties and their influence on inferred abundances and stellar evolution. While these "complications" are generally believed to be less prominent in Population II stars, they may still have a non-negligible impact on the quantitative picture.

As is commonplace in modelling of any kind, there are some mismatches between the run of inferred GC surface abundances and those predicted by stellar-structure models with atomic diffusion and mixing. However, given the tests that the atomic-diffusion hypothesis has been subjected to (using different spectrographs, different model atmospheres, different effective-temperature scales, different line-formation theories, different abundance-analysis techniques, spectral lines with different sensitivities to $T_{\text {eff }}$ and $\log g$ ), there can be little doubt that surface abundances of old, low-mass stars are an explicit function of time. Lithium happens to be one of the elements most affected by atomic diffusion. Surface depletions of $0.25-0.4$ dex are predicted by the current generation of 
sophisticated stellar-structure models ("Richard models") without an accompanying effect on well-observed elements like calcium or iron exceeding $\approx 0.1 \mathrm{dex}$. It is difficult to unambiguously establish the reality of such shallow abundance trends (cf. the case of NGC 6752 with a T6.2 mixing model). But even evidence from lithium-only studies is accumulating (Meléndez, these proceedings).

If we convince ourselves that the well-observed TOP-part of the Spite plateau lies at $\log \varepsilon(\mathrm{Li})=2.25$, then the atomic-diffusion corrected surface abundance of these stars (around $\log \varepsilon=2.5$ at $[\mathrm{Fe} / \mathrm{H}]=-2$ ) are in marginal $(1.5 \sigma)$ agreement with the currently favoured WMAP-calibrated primordial value of $2.72 \pm 0.06$ (Dunkley et al. 2009). There may, however, be biases both in the stellar-atmosphere, line-formation and stellarstructure modelling that could make the stellar abundances fall short. There may be remaining biases in the $\mathrm{BBN}$ predictions (cf. the relatively recent correction of the ${ }^{7} \mathrm{Be}(d, p) 2 \alpha$ cross-section at BBN energies, Angulo et al. 2005), even though few dare to say so (that would be PC blasphemy!). As an example of the remaining uncertainties of the stellar-atmosphere side, let us recall that an effective-temperature scale hotter by $100 \mathrm{~K}$ (as favoured by recent 3D Balmer-profile analyses, González Hernández et al. 2009 ) would add a further 0.07 dex to the above-mentioned valuest.

One may wish to add extra boundary conditions to the picture: the possibility of global Li-7 destruction by decaying super-symmetric particles early on in the Universe (indeed, during the very phase of BBN taking place on the natural time and energy scale for this to happen, cf. Jedamzik, these proceedings); the possibility of global Li-7 destruction by Population III stars (Piau et al. 2006); the observation of fragile Li-6 in some of the classical Spite-plateau halos stars which currently divides the hydro-modelling experts into believers and sceptics (Asplund et al. 2006, Cayrel et al. 2007; cf. the contributions by Asplund and Steffen, these proceedings); the possibility of global Li-6 production by the above-mentioned or a similar cosmological process (e.g. Jedamzik et al. 2006). All these make lithium a fascinating element to observe and model. But until solid evidence is provided for any of these effects, I prefer to settle for the physics we know (Principle of Parsimony): stellar structure and evolution as a remarkably precise theory. This does not free us of the need to develop this theory (and its atmospheric counterpart) to full radiation-hydrodynamic self-consistency.

\section{References}

Angulo, C., Casarejos, E., Couder, M., Demaret, P., Leleux, P., Vanderbist, F., Coc, A., Kiener, J., Tatischeff, V., Davinson, T., Murphy, A. S., Achouri, N. L., Orr, N. A., Cortina-Gil, D., Figuera, P., Fulton, B. R., Mukha, I., \& Vangioni, E. 2005, ApJ, 630, 105

Asplund, M., Lambert, D. L., Nissen, P. E., Primas, F., \& Smith, V. V. 2006, ApJ, 644, 229

Boesgaard, A. M., Deliyannis, C. P., Stephens, A., \& King, J. R. 1998, ApJ, 493, 206

Bonifacio, P. 2002, A\&A A, 395, 515

Bonifacio, P., Pasquini, L., Spite, F., Bragaglia, A., Carretta, E., Castellani, V., Centurin, M., Chieffi, A., Claudi, R., Clementini, G., D’Antona, F., Desidera, S., Franois, P., Gratton, R. G., Grundahl, F., James, G., Lucatello, S., Sneden, C., \& Straniero, O. 2002, A\&AA, 390, 91

Cayrel, R., Steffen, M., Chand, H., Bonifacio, P., Spite, M., Spite, F., Petitjean, P., Ludwig, H.-G., \& Caffau, E. 2007, A\&3A, 473, L37

Charbonnel, C. \& Zahn, J.-P. 2007, A\&A, 467, 15

Coc, A., Vangioni-Flam, E., Descouvemont, P., Adahchour, A., \& Angulo, C. 2004, ApJ, 600, 544

$\dagger$ If you are willing to bet a bottle of wine that the remaining 0.15-0.22 dex are due to new physics, contact the author. 
Deliyannis, C. P., Boesgaard, A. M., \& King, J. R. 1995, ApJ, 452, L13

Dunkley, J., Komatsu, E., Nolta, M. R., Spergel, D. N., Larson, D., Hinshaw, G., Page, L., Bennett, C. L., Gold, B., Jarosik, N., Weiland, J. L., Halpern, M., Hill, R. S., Kogut, A., Limon, M., Meyer, S. S., Tucker, G. S., Wollack, E., \& Wright, E. L. 2009, ApJS, 180, 306 González Hernández, J. I., Bonifacio, P., Caffau, E., Steffen, M., Ludwig, H.-G., Behara, N. T., Sbordone, L., Cayrel, R., \& Zaggia, S. 2009, A $\mathscr{G} A$, 505, L13

Jedamzik, K., Choi, K.-Y., Roszkowski, L., \& Ruiz de Austri, R. 2006, JCAP, 07, 007

Korn, A. J., Grundahl, F., Richard, O., Barklem, P. S., Mashonkina, L., Collet, R., Piskunov, N., \& Gustafsson, B. 2006, Nature, 442, 657

Korn, A. J., Grundahl, F., Richard, O., Mashonkina, L., Barklem, P. S., Collet, R., Gustafsson, B., \& Piskunov, N. 2007, ApJ, 671, 402

Lee, J.-W., Kang, Y.-W., Lee, J., \& Lee, Y.-W. 2009, Nature, 462, 480

Lind, K., Korn, A. J., Barklem, P. S., \& Grundahl, F. 2008, A\&A, 490, 777

Lind, K., Primas, F., Charbonnel, C., Grundahl, F., \& Asplund, M. 2009, A\& A, 503, 545

Michaud, G., Fontaine, G., \& Beaudet, G. 1984, ApJ, 282, 206

Pasquini, L. \& Molaro, P. 1996, A\&A, 307, 761

Pasquini, L., Bonifacio, P., Molaro, P., Francois, P., Spite, F., Gratton, R. G., Carretta, E., \& Wolff, B. 2005, A\& $A, 441,549$

Piau, L., Beers, T. C., Balsara, D. S., Sivarani, T., Truran, J. W., \& Ferguson, J. W. 2006, ApJ, 653,300

Piotto, G., Bedin, L. R., Anderson, J., King, I. R., Cassisi, S., Milone, A. P., Villanova, S., Pietrinferni, A., \& Renzini, A. 2007, ApJ, 661, L53

Richard, O., Michaud, G., \& Richer, J. 2001, ApJ, 558, 377

Spite, M. \& Spite, F. 1982, Nature, 297, 483 